\title{
La place de l'imagerie dans le développement clinique et pré-clinique des nouveaux médicaments dans les pathologies neurodégénératives
}

\author{
Wladimir Kawiecki ${ }^{1}$, Alexis Genin ${ }^{2}$, Alexandra Auffret ${ }^{2}$ et les participants de la table ronde $N^{\circ} 2$ de Giens XXVII* \\ 1 Siemens Healthcare, Saint-Denis, France \\ 2 ICM, Groupe hôspitalier Pitié-Salpétrière, Paris, France
}

Texte reçu le 20 mars 2012 ; accepté le 4 juin 2012

\author{
Mots clés : \\ imagerie ; recherche ; \\ développement ; \\ médicaments ; \\ maladies neurodégéné- \\ ratives
}

\begin{abstract}
Résumé - L'objectif de la table ronde était d'aborder la place de la neuroimagerie clinique et préclinique dans le développement de nouveaux médicaments dans les pathologies neurodégénératives pour la première fois lors des rencontres de Giens. Les participants venaient de de l'académie, du monde de la recherche clinique et fondamentale, de l'industrie du médicament et de l'imagerie, de l'Agence nationale de sécurité du médicament et des produits de santé (ex-Afssaps [Agence française de sécurité sanitaire des produits de santé]). Ils ont fait le constat de l'impact important de la neuroimagerie dans la connaissance des maladies neurodégénératives, en particulier la maladie d'Alzheimer et dans le développement des médicaments. Ils ont identifié les différentes approches techniques utilisées, les innovations à venir, des exemples à succès de travail en consortium mais également un risque trop important de ne pas pouvoir exploiter la neuroimagerie à tous les niveaux de la recherche. Dans ce contexte, la table ronde a conclu et a proposé différentes mesures pour renforcer la place méritée de l'imagerie.
\end{abstract}

Abréviations : voir en fin d'article.

\section{Le contexte}

Au cours des réunions préliminaires de cette table ronde, nous avons choisi de concentrer notre travail sur la place de la neuroimagerie dans le cadre de la recherche sur la maladie d'Alzheimer étant donné son impact socio-économique majeur.

Les coûts du développement de médicaments n'ont cessé d'augmenter avec en parallèle d'une baisse de productivité comme en témoigne l'augmentation des délais de mise sur le marché et la diminution du nombre d'autorisations délivrées en particulier dans le domaine des maladies neurodégénératives. Avec un besoin médical de plus en plus élevé, le développement de nouveaux médicaments dans le domaine des pathologies neurodégénératives se heurte à des barrières diverses, et en particulier dans le cas de la maladie d'Alzheimer, parmi lesquelles :

$1:$ l'absence de compréhension fine des mécanismes physiopathologiques de la maladie ;

2 : un diagnostic qui laisse peu d'espace d'intervention aux candidats médicaments à un stade où les dommages cérébraux sont déjà irréversibles ;
3: le manque de pertinence de l'appellation «maladie d'Alzheimer », qui englobe un ensemble de pathologies diverses ;

4 : le manque d'outils et de méthodes pour améliorer la prédictivité - efficacité et tolérance - des molécules en développement qui permettraient de cibler précisément leur potentiel, de diminuer leur temps de développement, d'améliorer l'efficience des études cliniques et de diminuer leurs durées ainsi que leurs coûts.

Le besoin est particulièrement important, car en raison du vieillissement de la population, le chiffre de 35,6 millions de cas devrait doubler d'ici 2050. ${ }^{[1]}$ À titre d'exemple, la recherche sur la maladie d'Alzheimer ces dernières années a généré tellement de connaissances qu'il serait difficile de les résumer, reflétant ainsi la complexité des maladies neurodégénératives. Cette complexité associée aux échecs des essais cliniques a soulevé des préoccupations suggérant que la communauté scientifique aurait peut-être cherché dans la mauvaise direction. ${ }^{[2]}$

Il existe toujours un besoin urgent de trouver des traitements efficaces contre la maladie d'Alzheimer. Les seules approches pharmacologiques disponibles et mises sur le marché sont des traitements symptomatiques, les inhibiteurs d'acétylcholinestérase et

\footnotetext{
* Pour la liste des participants, voir en fin d'article.
} 
un antagoniste des récepteurs glutamatergiques methyl-d-asparticacid (NMDA). Ces thérapies symptomatiques ont démontré des effets relativement modestes, ce qui a poussé la recherche vers le développement de molécules bloquant les processus pathologiques eux-mêmes (disease modifiers). Ces dernières approches se sont principalement portées sur les composés ciblant la voie -amyloïde. Il est également établi que d'autres mécanismes et voies moléculaires sont impliqués dans les processus pathologiques tels que la protéine tau, l'inflammation et d'autres processus qui joueraient un rôle important dans cette maladie multifactorielle. Plus particulièrement, le manque de marqueurs validés et efficaces reste un élément déterminant pour expliquer les échecs répétés dans le développement de médicaments candidats. L'utilisation et les qualités des marqueurs sont multiples : outil translationnel, outil de prédictivité, outil diagnostic, méthode de stratification des répondeurs et non-répondeurs.

La nature multifocale de la pathologie nécessite le recours à différents marqueurs cognitifs, d'imagerie, biologiques, génétiques, neurophysiologiques. Dans ce contexte, les attentes principales des entreprises du médicament sont aujourd'hui de disposer de nouveaux marqueurs qualifiés afin de concevoir des preuves de concept cliniques (phase II) sur un petit nombre de patients, sélectionnés à un stade de la maladie aussi précoce que possible et d'obtenir rapidement une indication de l'efficacité du candidatmédicament.

\section{Les promesses de la neuro-imagerie}

Les marqueurs d'imagerie émergent comme des outils particulièrement innovants, puissants et fiables pour répondre à ces attentes, et parmi eux essentiellement les marqueurs d'imagerie par résonance magnétique (IRM) et de tomographie par émission de positrons (TEP). L'IRM est une technique basée sur la résonance magnétique nucléaire de noyaux atomiques soumis à un champ magnétique extérieur. Cette technique permet de mesurer finement les volumes de certaines régions cérébrales, leur activité (grâce aux techniques d'IRM fonctionnelle) et leur connectivité (grâce à des modalités récentes comme l'imagerie de diffusion). La TEP est une imagerie fonctionnelle et moléculaire basée sur la détection des positrons. Le radioélement, le fluor 18 (F18) généralement, peut être chimiquement couplé à une molécule marquant spécifiquement certaines structures ou métabolites cérébrales et être détecté.

Deux types de marqueurs en imagerie peuvent être distingués. La première catégorie correspond à des marqueurs indépendants de la physiopathologie et pouvant être utilisés pour déterminer la topographie des lésions cérébrales (IRM avec analyses volumétriques, TEP-CT et la single photon computerized tomography [SPECT]). La seconde catégorie correspond à des marqueurs spécifiques des lésions de la pathologie (plaques séniles, dégénérescences neurofibrillaires, etc.) ou non-spécifiques (activation inflammatoire).
Dans le domaine de la maladie d'Alzheimer, plusieurs marqueurs de la plaque amyloïde comme le Pittsburgh compound B (PIB), le florbetaben et l' Avid 45 (AV45) sont maintenant utilisés dans les essais cliniques pour la sélection et le suivi des patients. Ces traceurs marqués au F18 seront destinés à être commercialisés et distribués au même titre que le FDG (fluorodésoxyglucose) comme produit de contraste. Par ailleurs, d'autres traceurs des dégénérescences neurofibrillaires (comme le 2-(1-\{6-[(2-[F18]Fluoroethyl)(methyl)amino]-2-naphthyl \}ethylidene)malononitrile [FDDNP], le Tau 808 [T808]) ou d'activation microgliale (marqueur PK1195) sont en cours de développement à des fins de recherche pour explorer différents mécanismes pathologiques de la maladie et pour des phases précoces de développement de nouvelles solutions thérapeutiques (phase $\mathrm{O}$ à II). ${ }^{[3]}$

\section{Des exemples à succès}

L'amélioration de la performance diagnostique offerte par les nouveaux équipements n'est cependant qu'une partie de la réponse à la question, une majeure partie de celle-ci résidant dans la puissance d'analyse des données d'imagerie, afin d'en extraire le signal et le résultat pertinent. Face à l'ampleur du défi, l'approche en réseau interdisciplinaire telle qu'elle est développée déjà aux États-Unis et en Europe est absolument indispensable au succès.

L'initiative Alzheimer's disease neuroimaging initiative (ADNi) a pour objectif de valider des marqueurs prédictifs de la progression de la maladie d'Alzheimer. L'objectif est également de travailler sur des équipements et traitements de données d'imagerie harmonisés. Les données recueillies sont mises à jour régulièrement et disponibles par le biais d'une plateforme. Cette initiative s'est étendue au niveau européen (projet IMI-PharmaCog) et mondial (Japan-ADNI, etc.). ${ }^{[4]}$ L'objectif de cette initiative visait à recruter 400 sujets atteints au début de déficience cognitive légère (mild cognitive impairment [MCI]), 200 sujets atteints de maladies d'Alzheimer, et 200 sujets témoins normaux. Soixante-sept millions de dollars de financement ont été fournis par les secteurs publics et privés, y compris le National Institute on Aging, 13 entreprises pharmaceutiques, et 2 fondations par le National Institutes of Health. Les principales réalisations de cette initiative ont été de combiner des méthodes alternatives de catégorisation de diagnostic où la neuro-imagerie joue un rôle majeur afin de développer les méthodes pour la détection précoce de la maladie jusqu'à des stades pré-symptomatiques, d'exploiter des mesures IRM qui sont plus prédictives du déclin cognitif que d'autres modalités. [5]

Une autre initiative coalition against major disease (CAMD) est à l'origine de la création d'une base de données contenant des informations sur 4000 patients Alzheimer inclus dans 11 essais industriels. Douze entreprises pharmaceutiques, 7 associations de patients, les grandes institutions de recherche et les autorités 


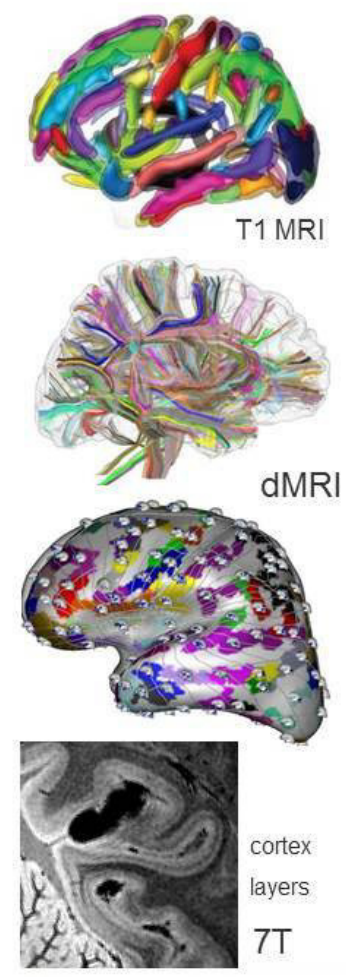

\section{CATI: a French Platform} dedicated to multi-center Studies in AD

Standardized MRI and PET Acquisitions

across a wide network of hospitals

\section{Centralized Quality control}

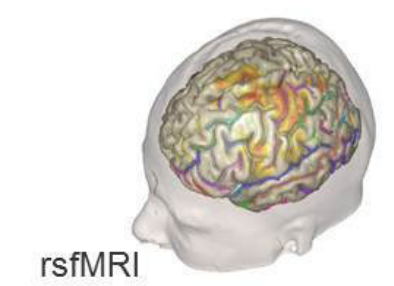

Portfolio of Image Analysis Pipelines

and machine learning techniques

for biomarker research

40 engineers and

clinical research assistants

9Meuro grant

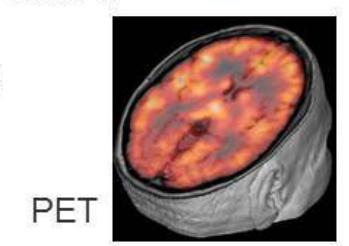

Fig. 1. Centre d'acquisition et de traitement de l'image (CATI).

L'objectif de ce centre consiste à mettre à disposition de la communauté scientifique et médicale travaillant sur la maladie d'Alzheimer, ces savoir-faire en assurant un traitement centralisé de l'image et des processus d'extraction de connaissance exploitables à partir de grands volumes d'information générées par un réseau d'appareils d'imagerie répartis sur l'ensemble du territoire français. Ce centre réunira un réseau d'équipes de recherche cliniques et fondamentalistes travaillant sur la maladie d'Alzheimer et maladies apparentées, équipées d'imageurs IRM et TEP dédiées à la recherche ainsi que des équipes de recherche en traitement d'images et en analyse de données travaillant sur la problématique Alzheimer.

règlementaires américaines participent à cet effort. ${ }^{[6]} \mathrm{Ce}$ consortium n'est pas financé pour réaliser des recherches mais pour exploiter des données déjà existantes. En octobre 2011, l'initiative CAMD a communiqué les premiers résultats de cette initiative en annonçant et proposant des nouveaux standards pour la recherche et le développement de médicaments pour la maladie d'Alzheimer. En parallèle, l'agence européenne des médicaments (EMA) et la Food and Drug Administration (FDA), ont annoncé de nouvelles directives pour le développement clinique. Traditionnellement, les chercheurs ont utilisé des tests de mémoire comme critère décisionnel. Il s'est avéré que ces tests à eux seuls n'étaient pas suffisamment sensibles en termes diagnostiques et d'évolution de la maladie. Ces dernières années, la combinaison d'autres biomarqueurs pourrait être beaucoup plus efficace comme la mesure en imagerie du volume hippocampique. En France, l'effort de structuration et de mise en réseau de la communauté a commencé dans le cadre du plan Alzheimer et des investissements d'avenir avec par exemple la création du Centre d'acquisition et de traitement de l'image (CATI) [figure 1], réseau national coordonné par le dipôle Neurospin/ICM (Institut du cerveau et de la moelle épinière) et avec l'initiative France Life Imaging. ${ }^{[7-8]}$ La France bénéficie par ailleurs d'une communauté de recherche au meilleur niveau international. Ces efforts de recherche en combinaison avec les nouvelles directives des autorités de santé renforcent la place de l'imagerie dans le développement clinique et pré-clinique des nouveaux médicaments.

\section{Un parc d'équipements d'imagerie au dernier rang europeen}

La France est vraisemblablement le seul pays où l'acquisition de ces appareils est réglementée pour un usage clinique (réglementation des « équipements lourds»). De plus, la France dispose en 2011 de moins de 10 IRM pour un million d'habitants ce qui la 


\section{IRM par million d'habitants en Europe de l'Ouest hors France (1/1/2009)}

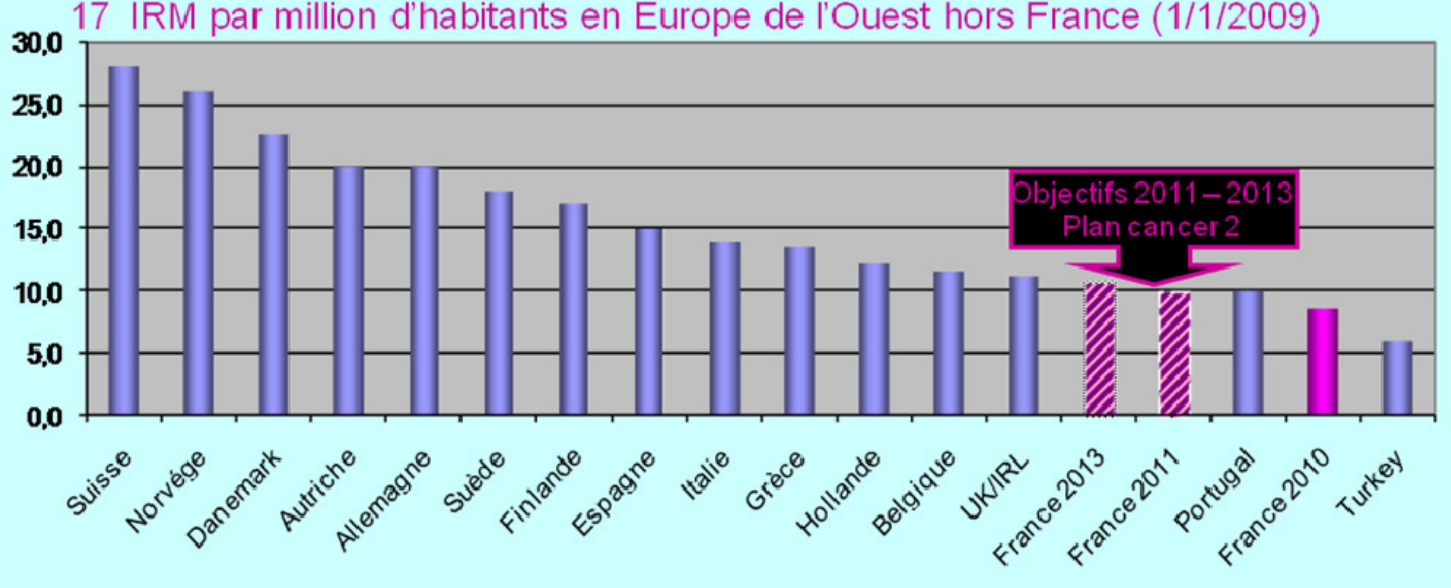

Fig. 2. Nombre d'IRM (clinique et recherche) par million d'habitants en Europe. La France dispose d'un parc imagerie trop faible avec moins de 10 IRM par million d'habitants. Les objectifs posés par le plan cancer $n^{\circ} 2$ n’ont guère amélioré la situation. Avec l'aimable autorisation de Imagerie Santé Avenir, Mme Marie-Hélène Coste, MHC Communication (rapport Annuel 2011).

IRM : imagerie par résonnance magnétique.

place en queue du peloton européen, derrière le Portugal et la Grèce et loin derrière l'Allemagne, la Norvège et la Suisse (figure 2).

Une récente étude de l'Agence nationale d'appui à la performance des établissements de santé et médico-sociaux (ANAP) a prouvé que la très grande majorité de ces équipements est intensivement utilisée avec des plages horaires élargies et un nombre de patients élevé. Le nombre d'appareils dont disposent les Centres hospitaliers universitaires (CHU) est relativement faible (moins de 3 par université) et le nombre de machines 3 Tesla (ayant un meilleur rendu en résolution spatiale - indispensable en neurologie) ne dépasse pas les $7 \%$. Dans une telle pénurie d'appareils cliniques, il est difficile de penser qu'il soit possible d'exploiter ces machines pour la recherche clinique impliquant des cohortes de malades. À ces 600 appareils cliniques se rajoutent un faible nombre de machines « hors carte sanitaire » (étant utilisées exclusivement pour la recherche, elles échappent à la réglementation d'autorisation). Ils sont au nombre d'une vingtaine (1,5 et $3 \mathrm{~T}$ ) couvrant tous les domaines de recherche auxquels se rajoutent un seul appareil de $7 \mathrm{~T}$ (à travers le monde une cinquantaine de sites de recherche utilisent déjà ce champ élevé) qui permet d'obtenir un gain très important en terme de sensibilité aux conséquences physiopathologiques de la maladie. La situation du parc IRM est celle d'une pénurie aussi bien en ce qui concerne les équipements de routine diagnostique que de recherche.

Le plan Alzheimer français prévoyait le financement de deux équipements IRM $7 \mathrm{~T}$ destinés exclusivement à la recherche sur la maladie d'Alzheimer. Cette ligne budgétaire a été modifiée à deux reprises, tout d'abord pour financer un nombre plus important de machines $3 \mathrm{~T}$ et finalement pour subventionner des examens IRM sur des machines déjà existantes.
En ce qui concerne les TEP, même si le parc français couvre globalement les indications de routine clinique en oncologie, leur nombre est insuffisant pour la recherche avec seulement 6 appareils destinés à la recherche, et pour la plupart anciens.

Les cyclotrons ou accélérateurs de particules permettent la production des isotopes radioactifs utilisés en médecine nucléaire. Les cyclotrons destinés exclusivement à la mise au point de radioisotopes de recherche ne sont qu'au nombre de 2 et ne se trouvent pas systématiquement intégrés directement dans les sites de recherche clinique et pré-clinique. Les cyclotrons dit de production sont en nombre suffisants pour la distribution commerciale du fluorodésoxyglucose (FDG) mais seulement trois sont dans une structure intégrée de recherche, les autres étant installés dans des zones industrielles.

Le F18 pourrait être livré sur tout le territoire français, mais ils n'existent que très peu d'équipes de recherche de radiochimie et radiopharmacie ayant des compétences dans l'association de molécules innovantes au F18 ou au C13 réalisée dans des conditions good manufacturing practices (GMP).

Il est également important de mentionner que la réglementation sur la production GMP est de plus en plus exigeante et en « mouvance constante » qui nécessite l'adaptabilité rapide des équipes.

Les équipements des différentes modalités en imagerie préclinique existent en faible nombre (une petite vingtaine de $\mu$ TEP et de RMN animale) et sont dispersées sur une quinzaine de sites de recherche. Ces machines sont principalement utilisées pour l'oncologie et la cardiologie environ à $90 \%$ et il n'existe que de très rares machines dédiées aux neurosciences sur le territoire. Dans le cadre d'une stratégie de recherche translationnelle, il est nécessaire d'augmenter le nombre d'équipements dédiés et de faciliter la 
répartition stratégique de ces machines pour proposer aux chercheurs tous les types d'outils d'analyses précliniques et cliniques sur un seul et unique site.

Plus récemment, de nouvelles offres technologiques avec une intégration MR\&PET en un seul appareil avec acquisition simultanée sont de nouvelles opportunités pour la recherche biomédicale. Un des enjeux sera donc de permettre l'acquisition rapide et suffisante de ce type d'équipement sur le territoire.

\section{Conclusions}

L'explosion des coûts de développement de médicaments peut être un vecteur de développement des techniques d'imagerie qui sont essentielles comme illustré précédemment : outil diagnostic plus sensible et résolutif, stratification des sujets, suivi des surrogate endpoints, transversalité en préclinique, possibilité de collaborations d'envergure. Certains aspects peuvent freiner son expansion : le cout élevé des équipements, la rentabilité des traceurs non remboursés, la nécessité de compétences pour l'extraction, l'harmonisation et l'interprétation d'une importante quantité de données, une expérience limitée des autorités réglementaires pour évaluer des nouveaux produits en provenance des techniques d'imagerie.

Sur le plan national, la place de l'imagerie dans le développement des nouveaux médicaments dans les pathologies neurodégénératives reste sous-estimée et un plan d'action doit être rapidement mis en place.

\section{Recommandations de la table ronde}

L'approche la plus productive pour le développement d'une neuroimagerie facilitant le développement de médicaments innovants serait le renforcement des centres d'excellence intégrés pour l'imagerie de recherche translationnelle, et la création de nouveaux centres. Ce type de structure présenterait le meilleur environnement pour une recherche efficace avec un plateau d'imagerie préclinique et clinique. Équipés d'IRM, de TEP (tomographie par émission de positions - tomodensidomètre [TEP-CT], tomographie par émission de position - magnetic resonance [TEP-MR]) et d'un cyclotron, ces centres devraient disposer d'équipes de recherche, y compris en radiopharmacologie, être localisés au sein d'un établissement clinique garantissant le recrutement de patients et regroupant les différentes compétences de spécialités cliniques.

Sans attendre de disposer comme certains autres pays de ce type de centre d'excellence, plusieurs actions semblent indispensables et peuvent être rapidement mises en œuvre :

1 : application de la mesure Nr 29 du Plan Alzheimer, c'est-àdire l'acquisition d'IRM $3 \mathrm{~T}$ dans les Centres mémoire de ressource et de recherche (CMRR) et d'autres sites de recherche ce qui permettra de renforcer le réseau des centres français en neuroimagerie. Une préférence pourrait être donnée aux machines plus récentes magnetic resonance-positron emission tomography (MRPET) qui permettront des acquisitions simultanées. Il serait également nécessaire d'établir un plan d'équipement d'IRM à très haut champs (7T) sur ces pôles d'imagerie de référence ;

2 : dynamisation de la filière française de $\mathrm{R} \& \mathrm{D}$ en radiochimie et radiopharmacie. Une approche simple serait de donner suite aux demandes formulées par la Société française de médecine nucléaire (SFMN) i.e., avec la mise en place d'une autorisation provisoire d'utilisation et de commercialisation pour évaluation clinique (APUCEC) ou du remboursement du médicament radiopharmaceutique indépendamment de l'examen TEP pour donner un cadre légal assoupli ainsi que des moyens financiers pour cette recherche de nouveaux médicaments radiopharmaceutiques ;

3 : renforcement et accroissement de la visibilité des réseaux nationaux autour des maladies neurodégéneratives. Structurer et augmenter la visibilité des réseaux de recherche à l'image du réseau CATI au niveau national ou d'autres consortiums internationaux seraient un excellent moyen de motiver les équipes de recherche et de pérenniser les échanges en neuroimagerie. Cela représenterait également un excellent modèle pour mutualiser les équipements, proposer des conditions de recherche multicentriques et harmonisées ce qui favoriserait les partenariats avec les acteurs industriels et permettrait d'envisager plus de partages de données ;

4 : renforcement de la communication avec les différentes autorités réglementaires. Une communication constructive devra permettre l'adaptation anticipative des contraintes réglementaires et d'éviter les échecs du passé. Par ailleurs, cette communication facilitera la qualification de la place de l'imagerie comme marqueur secondaire.

Dans la lignée des recommandations de la table ronde, plusieurs projets de recherche incluant l'imagerie préclinique et clinique ont été retenus dans les différents volets du programme « Grand emprunt - investissements d'avenir ». Parmi ces projets : l'Institut de neurosciences translationnelles de Paris (IHU-A-ICM) sur le site de la Pitié-Salpêtrière, l'Equipex «LILI » MR-PET à Lyon, l'équipement IRM 7T «AMI » à l'université d'Aix-Marseille ou encore « France LIFE imaging » qui rassemble dans une infrastructure coordonnée, plusieurs plateformes d'imagerie. Ces investissements d'avenir représentent un premier pas important mais il reste à espérer que d'autres projets majeurs se verront rapidement attribuer des fonds afin de stimuler la recherche et de permettre aux équipes françaises de neuroimagerie de se hisser au niveau des 10 meilleures équipes internationales.

\section{Participants.}

Olivier Blin (Laboratoire GlaxoSmithKline, Chine), Sarah Catoen (Laboratoire Guerbet), Catherine Deguines (ANSM, Paris), Nabil Bédira (Laboratoire Bristol Myers Squibb), Jean-Robert Deverre (Commissariat à l'énergie atomique, Saclay), 
Jean-Marie Goehrs (JMGoehrs Partners), Joëlle Micallef (CHU Hôpital de la Timone, Marseille), Bertrand Loubaton (GE Healthcare Europe), Jean-François Mangin (Commissariat à l'énergie atomique, Saclay), Pierre Payoux (Médecine nucléaire, CHU Purpan, Toulouse), Cyril Poupon (Comissariat à l'énergie atomique, Saclay; Neurospin), Jean-Philippe Ranjeva (CRMBM UMR CNRS, Faculté de Médecine, Aix-Marseille), Guy Turquet de Beauregard (IBA CIC BIO International SAS, Saclay), Nicolas Villain (Philips), Nadine Weisslinger (Laboratoire GlaxoSmithKline), Mohammed Zaim (Institut de Recherche Pierre Fabre).

\section{Conflits d'intérêts. Aucun.}

Abréviations. ADNi : Alzheimer's disease neuroimaging initiative; ANNP : Agence nationale d'appui à la performance des établissements de santé et médico-sociaux ; APUCAC : autorisation provisoire d'utilisation et de commercialisation pour évaluation clinique; AV45 : Avid 45; CAMD : coalition against major disease ; CATI : Centre d'acquisition et de traitement de l'image ; CHU : Centre hospitalier universitaire ; CMRR : Centre mémoire de ressource et de recherche ; EMA : Agence européenne du médicament ; FDA : Food and Drug Administration ; FDDNP : 2-(1$\{6-[(2-[F-18]$ Fluoroethyl)(methyl)amino]-2-naphthyl $\}$ ethylidene)malononitrile ; FDG : fluorodésoxyglucose ; GMP : good manufacturing practices ; IHU-A-ICM : Institut de neurosciences translationnelles de Paris ; IRM ; imagerie par résonnance magnétique; MCI : mild cognitive impairment; MR-PET : magnetic resonance- positron emission tomography; Neurospin/ICM :
Institut du cerveau et de la moelle épinière ; NMDA : methyl-daspartic acid ; PIB : Pittsburgh compound B; SFMN : Société française de médecine nucléaire ; SPECT : single photon computerized tomography ; T808 : Tau 808; TEP : tomographie par émission de positrons; TEP-MR : tomographie par émission de positonsmagnetic resonance.

\section{Références}

1. World Alzheimer Report 2010. http://www.alz.co.uk/

2. Mangialasche F, Solomon A, Winblad B, et al. Alzheimer's disease: clinical trials and drug development. Lancet Neurol 2010; 9(7): 702-16

3. Alzheimer Research Forum. http://www.alzforum.org/

4. www.pharmacog.org

5. Weiner MW, Veitch DP, Aisen PS, et al. The Alzheimer's disease neuroimaging initiative: a review of papers published since its inception. Alzheimers Dement 2012 Feb; 8(1 Suppl): S1-68

6. Barratt RA, Bowens SL, McCune SK, et al. The critical path initiative: leveraging collaborations to enhance regulatory science. Clin Pharmacol Ther 2012 Mar; 91(3): 380-3

7. http://www-dsv.cea.fr/dsv/instituts/institut-d-imagerie-biomedicale-i2bm/ services/neurospin-neurospin

8. http://icm-institute.org/

Correspondance et offprints: Alexis Genin, ICM-GH Pitié-Salpêtrière, 47 boulevard de l'Hôpital, 75001 Paris, France.

E-mail : Alexis.Genin@icm-institute.org 